\title{
LES MICROCARCINOMES PAPILLAIRES DE LA THYROIDE A PROPOS DE 37 CAS
}

\author{
S.GRITLI, K. KHAMASSI, A. LACHKHEM, M. GRAJA, S. TOUATI, Z. OUESLATI, A. EL MAY*, \\ F. BEN SLIMĖNE**, A. LADGHAM. \\ SERVICE DE CHIRURGIE CARCINOLOGIQUE CERVICOFACIALE ET ORL, \\ * SERVICE D'IMMUNO-HISTO-CYTOLOGIE \\ ** SERVICE DE MÉDECINE NUCLÉAIRE \\ INSTITUT SALAH AZAÏZ TUNIS
}

\begin{abstract}
RESUME
OBJECTIF: Etudier les caractéristiques cliniques, thérapeutiques et le profil évolutif des microcarcinomes papillaires de la thyroïde (MCP) afin d'établir une meilleure prise en charge de ces tumeurs.

MATERIEL ET METHODES: II s'agit d'une étude rétrospective comprenant 37 patients présentant un MCP de la thyroïde.

RESULTATS: L'âge moyen était de 38 ans et un sex-ratio de 0,15. L'examen clinique et l'échographie cervicale trouvaient respectivement un nodule isolé de diamètre $2 \mathrm{~cm}$ dans 89,1 et $70,2 \%$, et un goitre multinodulaire dans 10,9 et $29,8 \%$. 26 patients ont eu une loboisthmectomie dont un seul une totalisation thyroïdienne dans un $2^{\text {me }}$ temps. Les 11 patients restants ont eu une thyroïdectomie totale en 1 seul temps. Ceux-ci ont par ailleurs bénéficié d'une irathérapie à dose ablative. Aucune récidive ni décès n'ont été notés avec un recul moyen de 6 ans.

DISCUSSION: Dans notre série $94,5 \%$ des MCP étaient de découverte fortuite. L'attitude en peropératoire vis-à-vis de la glande thyroïde et des ganglions dépend de l'examen extemporané. Sa positivité impose une totalisation thyroïdienne associée à un geste ganglionnaire. Dans le cas contraire, aucun geste complémentaire ne parait justifié. Notre attitude rejoint celle de la majorité des séries dans la littérature.

CONCLUSION: En cas de diagnostic différé de MCP, la thyroïdectomie totale ne semble pas apporter de meilleurs résultats comparée à la loboisthmectomie.
\end{abstract}

MOTS CLES : Nodules thyroïdiens, thyroïdectomie, microcarcinome papillaire

OBJECTIVE: To study clinical, therapeutic, and evolutive characteristics of papillary thyroid microcarcinomas (PTMC) to establish a better attitude in the management of these tumors.

PATIENTS AND METHODS: We carry a retrospective study about 37 patients having a PTMC.

RESULTS: The mean age was 38 years and sex-ratio 0,15 . Clinical examination and cervical ultrasonography found respectively an isolated nodule with a diameter $2 \mathrm{~cm}$ in 89,1 and in $70,2 \%$, and a multinodular goiter in 10,9 and $29,8 \%$.

26 patients had loboisthmusectomy and only one a completion thyroidectomy. The 11 remaining patients had total thyroidectomy with iodine 131 ablation. A 6 years mean follow-up revealed no case of recurrence or death.

DISCUSSION: In our series $94,5 \%$ of PTMC was incidentally found. Peroperative management of thyroid gland and cervical lymph nodes depends on extemporaneous examination. If this one is positive, completion thyroidectomy with node sampling or dissection should be performed. In the opposite case, no other surgical procedure is carried out. This attitude joins that one of most series.

CONCLUSION: In case of delayed diagnosis of PTMC, total thyroidectomy doesn't seem to be superior to partial resection in term of results.

KEY WORDS : Thyroïd nodules thyroïdectomy, papillary microcarcinoma

\section{INTRODUCTION}

Les microcarcinomes papillaires de la thyroïde (MCP) sont des cancers différenciés du corps thyroïde dont la taille n'excède pas $1 \mathrm{~cm}$. Ces tumeurs se distinguent par leur mode de découverte souvent fortuit devant un nodule thyroïdien en apparence bénin. Les séries autopsiques réalisées à grande échelle rapportent des prévalences de MCP variant entre $6 \%$ [1] et $35,6 \%$ [2]. En Tunisie, cette prévalence est estimée à $4 \%$, y compris le type vésiculaire [14]. Jusqu'à $16 \%$ des pièces de thyroïdectomie totale pourraient renfermer ce type de lésion [3]. Il est important de signaler que certains MCP peuvent se montrer comme des tumeurs à fort potentiel agressif avec métastases ganglionnaires ou viscérales, ce qui contraste avec leur habituel bon pronostic [4].

Le but de ce travail est d'étudier les caractéristiques cliniques, thérapeutiques, et le profil évolutif des MCP, afin d'établir une meilleure prise en charge de ces tumeurs.

\section{MATÉRIEL ET MÉTHODES}

II s'agit d'une étude rétrospective à propos de 37 cas de MCP colligés dans notre service sur une période de 10 ans (Janvier 1995-Décembre 2004). Les cas de foyers de MCP associés à des macro carcinomes n'ont pas été inclus dans l'étude. Tous les patients ont bénéficié d'un examen ORL et général, d'un bilan thyroïdien et d'une échographie cervicale. La scintigraphie thyroïdienne n'était pas systématique. 
L'indication opératoire a été portée en présence d'au moins un nodule de diamètre supérieur ou égal à $2 \mathrm{~cm}$. Une loboisthmectomie ou une thyroïdectomie totale avec un examen anatomopathologique extemporané ont été pratiquées. Dans le cas où celui-ci est positif, un prélèvement ganglionnaire sus claviculaire et/ou un curage fonctionnel ont été réalisés.

Après avoir eu le résultat de l'anatomopathologie définitive, l'attitude était soit de se contenter d'une simple surveillance clinique pour les patients ayant eu une loboisthmectomie, soit de surveiller par le balayage cervicothoracique à l'iode (BCT) et le dosage de la thyroglobuline (TG) avec un complément de traitement par iode radioactif pour les patients ayant eu une thyroïdectomie totale.

\section{RESULTATS}

L'âge moyen des patients était de 38 ans (19-70) et le sexratio de $0,15(5 \mathrm{H}, 32 \mathrm{~F})$. Des antécédents familiaux de goitre ont été trouvées dans 2 cas. Le délai moyen entre l'apparition des signes fonctionnels et la première consultation était de 3 ans (1mois-20 ans).

30 patients consultaient pour une tuméfaction basicervicale isolée, 3 pour des signes de compression associés (dysphagie aux solides et dyspnée d'effort), 3 pour des signes d'hyperthyroïdie associés. Un seul patient avait un goitre découvert lors d'un examen systématique.

L'examen clinique trouvait un nodule isolé dans 33 cas et un goitre multinodulaire dans 4 cas.

Le siège des nodules était lobaire dans 34 cas et isthmique dans 3 cas. Le caractère dur des nodules était trouvé chez 1 patient. Des adénopathies jugulocarotidiennes moyennes droites étaient présentes chez un autre patient. L'échographie cervicale trouvait un nodule isolé dans 26 cas et un goitre multinodulaire dans 11 cas dont un associé à des adénopathies cervicales.

Le bilan hormonal trouvait une hyperthyroïdie chez 2 patients. La scintigraphie thyroïdienne faite dans 30 cas trouvait une hypofixation dans 26 cas et une hyperfixation dans 4 cas.

26 patients ont eu une loboisthmectomie, l'examen extemporané revenait "bénin» dans 21 cas et «douteux» dans 5 cas. L'histologie définitive montrait la présence d'un MCP dans tous les cas. Une simple surveillance clinique a été préconisée chez 25 cas, alors qu'un seul patient a eu une totalisation thyroïdienne avec un curage médiastino-récurrentiel et prélèvements sus-claviculaires bilatéraux (anatomopathologie: "négatifs").

Les 11 patients restants ont eu une thyroïdectomie totale (Figure 1,2), l'examen extemporané revenait «bénin» dans 7 cas et "douteux" dans 1 cas. Celui-ci a eu un curage médiastino-récurrentiel (négatif) et fonctionnel droit devant la présence d'adénopathies (5 ganglions envahis avec rupture capsulaire, 20 ganglions négatifs), avec un prélèvement sus-claviculaire controlatéral (négatif). Un MCP a été trouvé à l'examen extemporané dans 3 cas, un prélèvement ganglionnaire sus-claviculaire bilatéral a été alors effectué et est revenu négatif. Dans les 11 cas, l'anatomopathologie définitive trouvait un MCP. Le BCT trouvait une fixation cervicale chez tous ces patients et une élévation de la TG chez 9 d'entre eux. En moyen- ne 2 cures d'iode radioactif à dose ablative (100 mCi) ont été administrées chez les 11 patients.

L'évolution a été favorable chez les 37 patients. Aucun cas de récidive n'a été noté avec un recul moyen de 6 ans (2 à 8 ans).

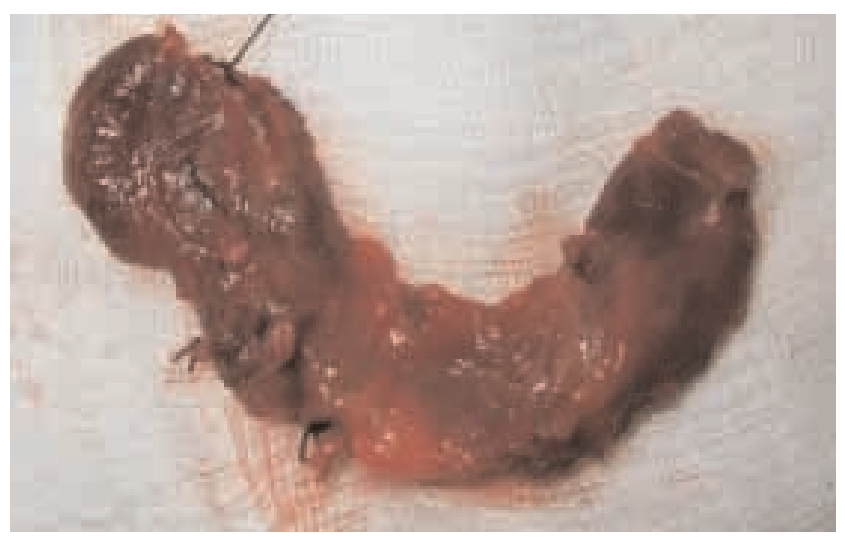

Fig. 1 : Pièce de thyroïdectomie totale

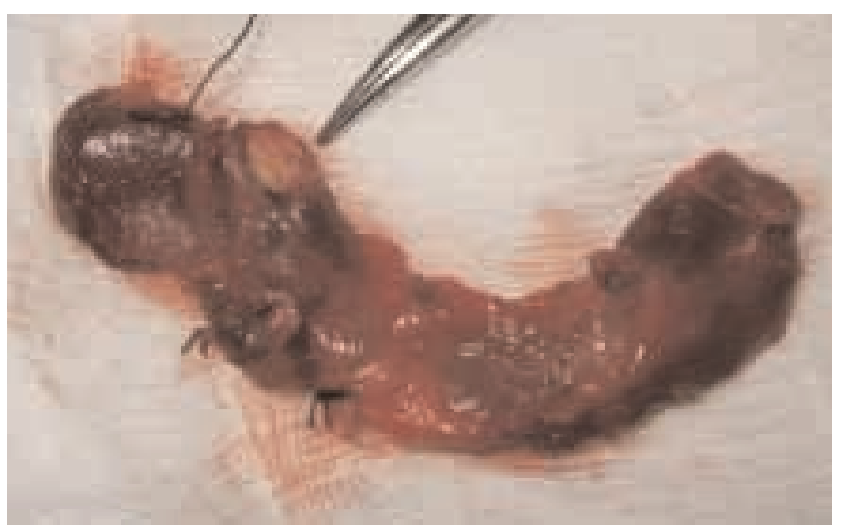

Fig. 2 : Aspect macroscopique de MCP

\section{DISCUSSION}

La discussion en matière de MCP s'oriente en fonction des circonstances du diagnostic. Celles-ci se résument en 3 situations.

La découverte par métastase ganglionnaire cervicale est classique mais peu fréquente [5] (3\% dans notre série). Devant ce potentiel invasif, une chirurgie standardisée de carcinome différencié de la thyroïde sera réalisée : thyroïdectomie totale associée à un prélèvement ou à un curage ganglionnaire cervical.

La découverte d'un MCP sur un nodule suspect en préopératoire est exceptionnelle, puisque par définition les MCP sont habituellement non palpables et qu'il est rare de retenir une indication chirurgicale sur de si petits nodules [5]. La prise en charge est la même que la précédente.

Les MCP sont souvent de découverte fortuite après une intervention pour un nodule ou un goitre multi nodulaire apparemment bénin [6] (94,5\% dans notre série). Dans ce cas, l'attitude en peropératoire vis-à-vis de la glande thyroïde et des ganglions cervicaux est étroitement liée au résultat de l'examen extemporané. Le manque de sensibilité de cet examen nous a amené à se contenter d'une 
loboisthmectomie, et après le résultat anatomopathologique définitif, un seul patient a eu une totalisation avec curage, les 25 autres ont eu une simple surveillance clinique. Pour les patients ayant eu une thyroïdectomie totale d'emblée, un prélèvement sus claviculaire a été fait en cas d'examen extemporané montrant un MCP. Dans le cas contraire, aucun geste ganglionnaire n'a été pratiqué même après le résultat définitif. Tous les patients ayant eu une thyroïdectomie totale ont eu une irathérapie à dose ablative.

A long terme, aucune récidive ni décès ne sont retrouvés dans notre série avec un recul moyen de 6 ans. Dans la littérature, le taux de récidive varie entre $0[15], 0,5$ [7] et $6 \%$ [8], le taux de décès ne dépasse pas $0,4 \%$ [8].

Pour notre part, l'attitude thérapeutique la plus adéquate est qu'une découverte peropératoire d'un MCP implique au minimum une totalisation thyroïdienne associée à un prélèvement ganglionnaire sus claviculaire bilatéral, et à un curage médiastino-récurrentiel bilatéral vu l'incidence élevée de l'atteinte des ganglions pré trachéaux dans les MCP [6].

Le curage fonctionnel est réservé aux patients ayant des adénopathies métastatiques [9] ou chez lesquels le prélèvement ganglionnaire revient positif. Cette attitude permettra surtout d'éviter la réintervention. Par la suite il est possible de suivre le taux de TG, de réaliser un contrôle scintigraphique et éventuellement une irathérapie dans les meilleures conditions.

En cas de découverte d'un MCP à l'analyse définitive de la pièce opératoire, faut-il réintervenir pour réaliser une totalisation et éventuellement un geste ganglionnaire ? Les deux attitudes se défendent puisque les auteurs qui ont le plus d'expérience dans ce domaine sont partagés entre un traitement maximaliste systématique ou seulement en cas de facteurs de risque. Pour Chow SM et al [10], malgré leur excellent pronostic, les MCP sont associés à un taux de mortalité de $1 \%$, à une récidive gan- glionnaire dans $5 \%$ des cas, à des métastases à distance dans $2,5 \%$ des cas ; par conséquent son traitement ne doit pas être différent de celui des macrocarcinomes : c'est-à-dire une chirurgie complète indépendamment de la taille de la tumeur. Pour Hay et al [11], les métastases, l'âge, l'étendue de la résection, le degré d'envahissement et la taille de la tumeur sont des facteurs de mauvais pronostic ; et la présence de tels facteurs impose un traitement complet [12] et une irathérapie à dose ablative $[10,12]$. Pour Garrel et al [5], aucun geste complémentaire n'a été réalisé dans sa série, un recul moyen de 7 ans a montré une seule récidive sur 64 cas de MCP (soit $1,56 \%$ ) contrôlée par irathérapie. Pour notre part, dans le cas des loboisthmectomies notre attitude était la surveillance dans la quasi totalité des cas, et aucune récidive n'a été notée sur un recul moyen de 6 ans.

La thyroïdectomie totale ne semble donc pas apporter de meilleurs résultats sur le plan pronostic comparée à la loboisthmectomie.

\section{CONCLUSION}

Bien que les MCP peuvent être agressifs et d'évolution rapide [13], ils sont généralement d' excellent pronostic $[5,9,15]$. Deux groupes de MCP s'opposent. Ceux qui sont découverts sur adénopathie doivent être traités de manière conventionnelle comme tout cancer différentié du corps thyroïde. Ceux qui sont découverts fortuitement à l'occasion d'une thyroïdectomie totale ou partielle sont à priori peu agressifs mais imposent l'analyse des autres critères pronostiques. La réalisation d'une totalisation thyroïdienne et d'un geste ganglionnaire est envisageable lorsque le diagnostic est porté durant l'intervention initiale. En cas de diagnostic différé, une réintervention pour totalisation et curage ganglionnaire ne parait pas justifiée en l'absence de facteurs de mauvais pronostic.

\section{REFERENCES}

[1] Lang W, Borrusch H, Bauer L. Occult carcinomas of the thyroid. Evaluation of 1020 sequential autopsies. Am J Clin Pathol 1988:90:72-76.

[2] Harach HR, Franssila KO, Wasenius VM. Occult papillary carcinoma of the thyroid. A 'normal' finding in Finland. A systematic autopsy study. Cancer 1985;56:531-538

[3] Yamashita $H$, Noguchi $S$, Watanbe $S$, Uchino $S$, Kawamoto $H$, Toda $M$ Murakami N, Nakayama I. Thyroid cancer associated with adenomatous goiter: an analysis of the incidence and clinical factors. Surg Today 1997:27:495-499.

[4] Leprat F, Trouette H, Cochet C, Saumthally B, Masson B, De Mascarel A Latapie JL. Papillary microcarcinoma of the thyroïd. Ann Endocrinol (Paris) 1994;54:343-346.

[5] Garrel $R$, Cartier C, Marvaso V Corpelet D, Markeieff M, Crampette L, Guerrier B. Notre expérience des microcarcinomes papillaires de la thyroïde. Rev Loaryngol Otol Rhinol $2002 ; 123,4: 239-242$.

[6] Nobuyuki W, Quan-Yang D, Kiminori S. Lymph node metastasis from 259 papillary thyroid microcarcinomas : frequency, pattern of occurrence and recurrence, and optimal strategy for neck dissection. Ann Surg 2003:237:399-407.

[7] Nogushi SYH, Murakami N, Nakayama I, Toda M, Kawamoto H. Small carcinomas of the thyroid. A long-term follow-up of 867 patients. Arch Surg 1996;131:187-191.

[8] Hay ID, Grant CS, Van Heerden JA, Goellner JR, Ebersold JR, Bergstralh EJ. Papillary thyroid microcarcinoma: a study of 535 cases observed in a 50-year period. Surgery 1992;112:1139-1146; discussion 1146-7

[9] Orsenigo E, Beretta E, Fiacco E, Scaltrini F, Veronesi P, Invernizzi L, Gini P,
Fiorina P, Di Carlo V. Management of papillary microcarcinoma of the thyroid gland. Eur J Surg Oncol 2004;30:1104-1106.

[10] Chow SM, Law SCK, Chan JKC, Au SK, Yau S, Lau WH. Papillary microcarcinoma of the thyroid-Prognostic significance of lymph node metastasis and multifocality. Cancer 2003;98:31-40.

[11] Hay ID, Bergstralh EJ, Geollner JR, Ebersold JR, Grant CS. Predicting outcome in papillary thyroid carcinoma: development of a reliable prognostic scoring system in a cohort of 1779 patients surgically treated at one institution during 1940 through 1989 . Surgery 1993;114:1050-1058

[12] Lin JD, Chen ST, Chao TC, Hsueh C, Weng HF. Diagnosis and therapeutic strategy for papillary thyroid microcarcinoma. Arch Surg 2005;140:940-945.

[13] Allo M, Christianson W, Koivunen D. Not all 'occult' papillary carcinomas are 'minimal'. Surgery 1988:104:971-976.

[14] Gritli S, Mnejja M, Khedim A, Marrakchi R, Ben Romdhane K. Le carcinoma occulte de la thyroïde: Etude autopsique à propos de 100 cas. J Tun ORL 2005; $\mathrm{N}^{\circ} 14$

[15] Oueslati Z, Aloui M, Gritli S, Touati S, El May A, Gamoudi A, Ben Slimène F, Ladgham A. Microcarcinomes papillaires de la thyroïde. Experience de l'Institut Salah Azaïz. Rev Laryngol Otol Rhinol $2002 ; 123,1: 39-42$. 